\title{
Patch pipettes are more useful than initially thought: simultaneous pre- and postsynaptic recording from mammalian CNS synapses in vitro and in vivo
}

\author{
Bert Sakmann
}

Received: 15 September 2006 / Accepted: 15 September 2006 / Published online: 10 October 2006

(C) Springer-Verlag 2006

Keywords Patch pipette. CNS synapses .

Thalamocortical pathway Calyx of Held

\section{Introduction}

Patch pipettes were designed, initially, for the purpose of recording ionic currents through individual channels in cell membranes using a single pipette placed on a muscle membrane [28]. Here, we could watch a single biological molecule as it changed its structure by opening and closing a transmembrane pore. For example, at acetylcholine (ACh) concentrations of about $1 \mu \mathrm{M}$ or higher, the same molecule switches repeatedly between an open and several closed state(s) [32]. The formation of a $\mathrm{G} \Omega$-seal between pipette tip and membrane widened the field of ion channel research. Currents were recorded with submillisecond resolution to resolve short opening and closing events [11]. Combined with genetic manipulation of ion channels expressed heterologously in Xenopus laevis oocytes, this technique facilitated the first structure-function assignments of ion channels. Single amino acids in putative pore-forming domains could be exchanged, and the subsequent effect on ion flows could be directly measured [20]. Finally, current recordings could be made from cell compartments such as the dendrite and terminal of a nerve cell.

B. Sakmann $(\bowtie)$

Department of Cell Physiology,

Max-Planck-Institute for Medical Research,

Jahnstr. 29,

69120 Heidelberg, Germany

e-mail: bert.sakmann@mpimf-heidelberg.mpg.de
"Patch pipettes are, however, more useful than initially thought." (Fred Sigworth) The whole-cell recording configuration also opened up a new field of research into the synapses of the mammalian central nervous system (CNS). In conjunction, the development of novel in vitro brain slice preparations and new visualization techniques in slices [12, 36] allowed us to characterize the properties of synapses in anatomically identified CNS pathways. Most useful were simultaneous whole-cell recordings made from cell pairs under visual control in combination with post hoc reconstructions of dendrites and axons of the two cells [26]. I will review the results we obtained by simultaneous pair recordings from pre- and postsynaptic CNS neurons and anatomical reconstructions. Each of the synapses we studied has a different function, and each is favorable to examine one particular aspect of synaptic function.

One question relating to the properties of synapses in a neuronal network is how they might contribute to the magnitude and variability of AP responses observed in sensory systems. Unit recordings from single cells in sensory cortices indicate large differences both in the strength (efficacy) and trial-to-trial variability of suprathreshold responses. The contribution of synapses to this variability in particular is, so far, not well understood. By comparing three different synapses that are components of sensory pathways, we illustrate how one might be able to identify their contribution to the reliability of sensory representations. The rationale of this approach is to compare in vivo the magnitude and variability of response to sensory stimuli at different stages of a pathway. Concomitantly, a particular synapse between two stages is studied in vitro to delineate factors that determine synaptic efficacy and reliability.

We first studied in vitro a relay synapse in the acoustic pathway. Cell pairs form an axosomatic synapse where the 
nerve terminal (the calyx of Held) is in direct contact with the postsynaptic cell soma. Here, the tips of the two recording pipettes are separated by less than $10 \mu \mathrm{m}$, and both can be viewed and manipulated under visual control. Secondly, also in vitro, we studied a local circuit synapse in the neocortex where the tips of the two pipettes that are used to record from pre- and postsynaptic cells in the neocortex are separated by $\approx 200-1,000 \mu \mathrm{m}$. Here, the field of view has to be changed for visualization of pipette tips when attaching one of the two pipettes to a soma of a pair. This necessitated the development of new, movable microscope stages for visualization and micromanipulation of pipette tip positions under visual control. In contrast, recordings to the calyx preparation are restricted to the two somata of pre- and postsynaptic cells, meaning that the synaptic contacts are located electrically remote from the recording pipette tips. Thirdly, more recently, we characterized in vivo a synapse in the thalamocortical (tc) pathway. This pathway "couples" the afferent somatosensory system to the neocortex. Here, the tips of the pair of patch pipettes are separated by several millimeters and (so far) cannot be visualized during the experiment. Finding a monosynaptic connection between the ventral posterior nucleus of the thalamus (VPM) and cortex relies upon topographically aligning the recording pipettes in the two brain regions and on a high density of individual tc axons and postsynaptic cortical dendrites.

\section{Results}

Relay synapse between two auditory nuclei in the brain stem The synapse between the calyx of Held and the soma of a medial nucleus of the trapezoid body (MNTB) neuron was anatomically described as part of the acoustic pathway by Held [18] (Fig. 1a,b). The synapse is a component of a long-range circuit between the inner ear and a nucleus in the brainstem that ensures precise timing of interhemisphere inhibition that enables localization of sounds. An auditory stimulus (e.g., a click) evokes a stereotyped AP response in this part of the acoustic pathway [29]. It is able to follow clicks with precisely timed APs even when they are presented at short intervals. To study efficacy of synaptic transmission between the globular bushy cells in the anteroventral cochlear nucleus (AVCN) and the principal cells in the MNTB (Fig. 1b), we prepared acute brainstem slices containing the calyx of Held and principal cells in the MNTB according to Forsythe [15]. Dual simultaneous recording under visual control and simultaneous $\mathrm{Ca}^{2+}$ fluorescence measurements were then established by Borst and Helmchen (Fig. 1c).

The AVCN-MNTB synapse has a high efficacy by which a single presynaptic AP evokes a large (unitary)
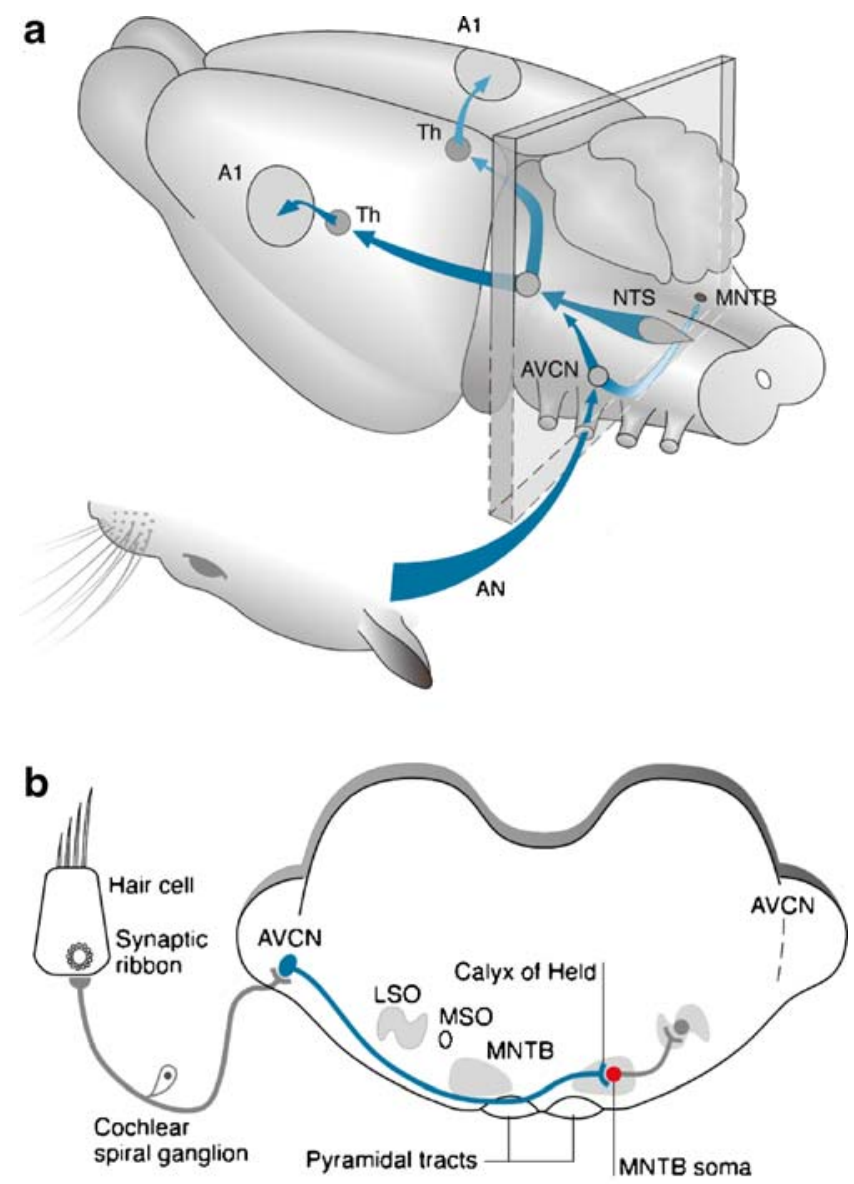

C
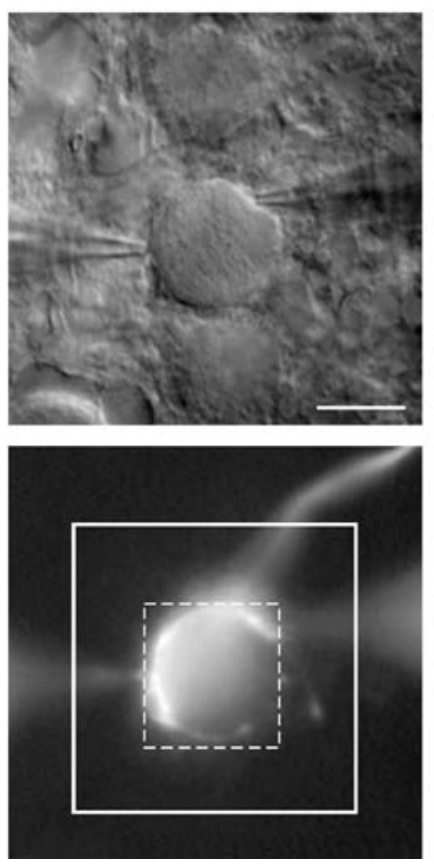

suprathreshold excitatory postsynaptic potential (EPSP) that rapidly triggers a postsynaptic AP (Fig. 2a). Estimates of the number of release sites made anatomically ([33], Fig. $2 b$ ) or functionally suggest $\approx 600$ synaptic contacts 
Fig. 1 Synaptic transmission between two nuclei of the rat auditory system. a Schematic illustration of the afferent connections in the acoustic system. The pathway from AVCN to MNTB contributes to the location of sounds by binaural hearing. $A N$ Acoustic nerve, $A V C N$ anteroventral cochlear nucleus, $M N T B$ medial nucleus of the trapezoid body, $A 1$ acoustic cortical area, Th thalamus. Cutting plane of acute brain-stem slice is indicated schematically. b Detailed schematic view of AVCN-to-MNTB pathway (blue) indicating the location of the calyx of Held terminal of globular bushy cells in the AVCN and the soma of MNTB principal neurons (red). LSO Lateral superior olive, MSO medial superior olive. c IR-DIC view of a calyx of Held-MNTB principal cell body in a living acute brain slice (upper panel). Same brain slice seen in fluorescence microscopy after filling calyx of Held with a fluorescent $\mathrm{Ca}^{2+}$ indicator (lower panel). The calyx recording pipette is seen on the left side, while the MNTB somatic recording pipette is seen on the right side. Squares indicate the area from which $\mathrm{Ca}^{2+}$ fluorescence signals are recorded from (see Fig. 2). Scale bar represents $10 \mu \mathrm{m}[19]$

[27]. The large number of synaptic contacts made by a single calyx is one factor that contributes to the high efficacy and reliability of this synapse. To dissect the other factors that lead to the high precision of AP transmission, one has to determine quantal content of a unitary EPSP and quantal size [31]. The quantal content is about 200 suggesting release of glutamate from 200 vesicles. Comparing this with the number of all, anatomically measured, synaptic contacts $\left(n_{\mathrm{a}}\right)$ made by a calyx (Fig. $2 \mathrm{~b}$ ), one finds that only about one-third of all synaptic contacts are releasing a vesicle upon an AP. Mean EPSC-variance analysis indicated that the release probability at the active zone (AZ) of each synaptic contact is relatively low, about $p_{\mathrm{r}}=0.25-0.4$ [27, see also 10] being responsible for the observation that only a fraction of all AZs releases transmitter during an AP.

The major determinant of reliable and phase-locked synaptic transmission is the time course of release probability, which in turn is governed by the size and time course of the local $\left[\mathrm{Ca}^{2+}\right]$ transient at vesicular release sites, operationally defined as the $\mathrm{Ca}^{2+}$ sensor. As the spatial relationship between the vesicle release sites, the $\mathrm{Ca}^{2+}$ entry sites, and the endogenous $\mathrm{Ca}^{2+}$ buffers is uncertain, the $\left[\mathrm{Ca}^{2+}\right]_{\mathrm{i}}$ dynamics at the site of the $\mathrm{Ca}^{2+}$ sensor were also unknown. We established recording conditions [4] that allowed us, eventually [2], to determine the time course and amplitude of the local $\left[\mathrm{Ca}^{2+}\right]_{i}$ transient during an AP in the close vicinity of a vesicular release site (Fig. 2e). We estimated the size and the time course of the local $\left[\mathrm{Ca}^{2+}\right]_{i}$ transient initially by back-calculating of the $\left[\mathrm{Ca}^{2+}\right]_{\mathrm{i}}$ transient during an AP using the experimentally determined kinetic parameters of the putative $\mathrm{Ca}^{2+}$ sensor that triggers release (Fig. 2c; [3, see also 34]). However, it remained unclear whether very short transient $\left[\mathrm{Ca}^{2+}\right]_{i}$ elevations were sufficient to trigger release similar to that observed during an AP. We mimicked the $\left[\mathrm{Ca}^{2+}\right]_{\mathrm{i}}$ transient during an AP by shaping the time course and amplitude of the $\left[\mathrm{Ca}^{2+}\right]_{i}$ transient evoked by an intracellular UV photolyzable $\mathrm{Ca}^{2+}$ cage via a UV flash. Shaping the transient was achieved by including a small amount of nonphotolyzable $\mathrm{Ca}^{2+}$ buffer in the presynaptic pipette solution. The EPSC in response to a short $\left[\mathrm{Ca}^{2+}\right]_{\mathrm{i}}$ transient is comparable in size and time course to that evoked by a single AP when the duration of the $\left[\mathrm{Ca}^{2+}\right]_{\mathrm{i}}$ transient is appropriately shaped (Fig. 2d; [2]). This measurement is in reasonable agreement with the initial back-calculated time course (Fig. 2c).

The local $\left[\mathrm{Ca}^{2+}\right]$ transient at the release site of AZs (Fig. 2e) evoked by an AP lasts for $<500 \mu$ s with a peak of 15-20 $\mu \mathrm{M}$ at the putative $\mathrm{Ca}^{2+}$ sensor that acts as a $\mathrm{Ca}^{2+}$ concentration follower. The duration of high release probability is very short due to the short $\mathrm{Ca}^{2+}$ current. The high sensitivity of release to the time course of the $\left[\mathrm{Ca}^{2}\right.$ $\left.{ }^{+}\right]_{i}$ transient may likely be a mechanism by which the waveform of the presynaptic AP modulates the release probability $\left(p_{\mathrm{r}}\right)$ and thereby synaptic efficacy.

Local circuit synapse between two layers of neocortex The representation of a tactile stimulus applied to a whisker on a rodent's face in the brain is almost punctate, being restricted largely to a small patch (single cortical column) of somatosensory cortex [37]. This precise topography is due to the relatively discrete arrangement of the connections between the follicle of a facial vibrissa and the somatosensory cortex via the trigeminal ganglion and the trigeminal brainstem nucleus (Fig. 3a). The tc projections from a single barreloid in the VPM (thalamic nucleus) terminate mostly within the outlines of a single column in the granular layer (layer 4; L4), the major recipient layer of cortex for sensory afferents (Fig. 3b). From the granular layer, the excitation is then conveyed, mostly within the boundaries of a single column, in the vertical direction to both supra- (Fig. 3b) and infragranular layers, L2/3 and L5, respectively [24]. The representation of a whisker deflection in $\mathrm{L} 2 / 3$ is sparse. Unbiased recording in vivo from individual cells in the upper layer of cortex [25] indicates that the response is low ( $<1 \mathrm{AP} /$ stimulus, [8]), and trial-totrial variability of AP responses is high with a coefficient of variation (C.V.) of $>1$ (de Kock et al., submitted). The local axonal projections of the recipient L2/3 cells are mostly in the tangential direction, and they supposedly integrate sensory signals from several whiskers. The long-range projections are mostly to other areas of the cortex suggesting that L2/3 cell activity eventually contributes to whisking and perceptual decision-making based on tactile input.

A source of the sparse AP representation and variability could be the efficacy of the synapse in single L4-L2/3 connections of this sensory pathway. The efficacy of a synaptic connection is as in the case of the AVCN-MNTB 
Fig. 2 Local $\left[\mathrm{Ca}^{2+}\right]_{\mathrm{i}}$ transient that determines precise timing in the AVCN-MNTB pathway. a Simultaneous pre- and postsynaptic recording from calyx and MNTB soma. Upon stimulation of AVCN axon, presynaptic (pre) AP is followed by EPSP (initial upstroke of tracelabeled post), triggering with short latency a postsynaptic AP [6]. b View of presynaptic face of a calyx (yellow) from serial EM reconstructions. Locations of active zones are indicated in red [33]. c Presynaptic AP (black trace) and back-calculated local $\left[\mathrm{Ca}^{2+}\right]_{\mathrm{i}}$ transient at vesicular release site (red trace) and vesicle release rate during an AP [3]. d $\left[\mathrm{Ca}^{2+}\right]_{\mathrm{i}}$ fluorescence transient (red trace) evoked by release of intracellular $\mathrm{Ca}^{2+}$ from a $\mathrm{Ca}^{2+}$ cage by a UV flash (arrow). $\left[\mathrm{Ca}^{2+}\right]_{\mathrm{i}}$ transient evokes an EPSC similar to AP-evoked EPSC ( $I_{\text {post }}$, lower trace). $\left[\mathrm{Ca}^{2+}\right]_{\mathrm{i}}$ transient

$\left(\Delta\left[\mathrm{Ca}^{2+}\right]_{\mathrm{i}}\right)$ was back-calculated from fluorescence transient $(\Delta \mathrm{F} / \mathrm{F})$ (black upper trace) [2]. e Two examples of active zones (AZs) of the calyx with docked vesicles (asterisks). Scale bar represents $0.1 \mu \mathrm{m}$ [33]

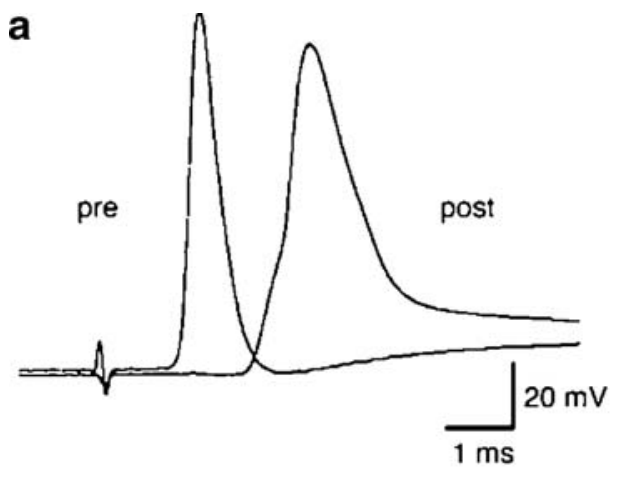

b

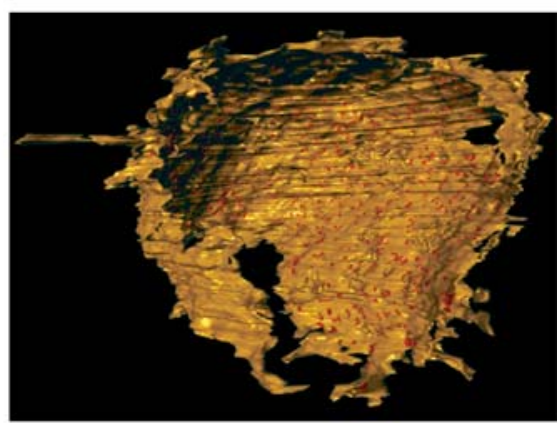

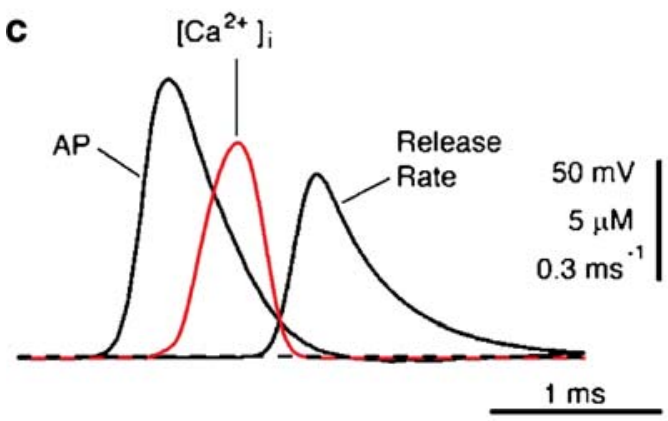

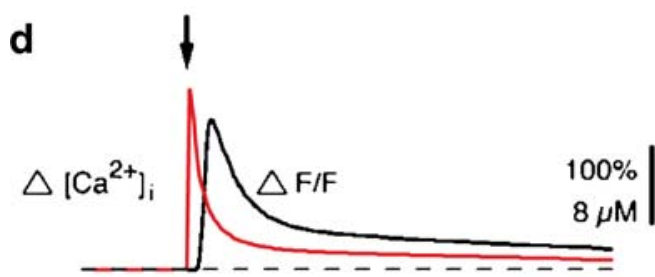

e
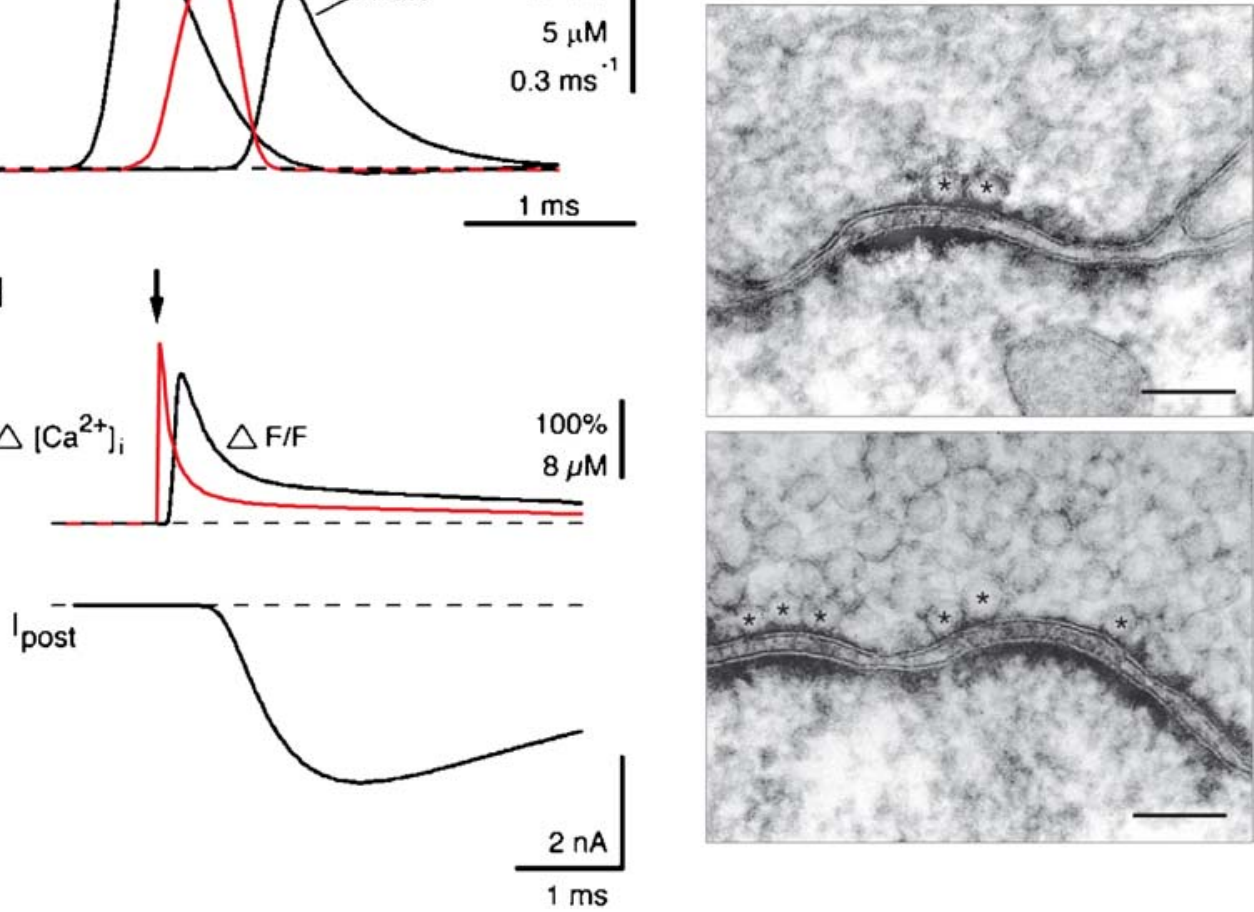

connection dependent on the number of release sites, the probability of release, and the size of the quantal response. The possible contribution of these factors to tactile stimulus response measured in vivo can be delineated with simultaneous pair recordings from pre- and postsynaptic cells in vitro. Feldmeyer used neocortex slices [1], allowing visualization of individual columns and making simultaneous recordings from cells located in the granular and supragranular layers (Fig. 3c). We found that the average size of unitary EPSPs, as a measure of synaptic efficacy, is small, having a range of $0.1-2 \mathrm{mV}$ when recorded at the soma of the L2/3 pyramid cell ([35], Fig. 4a; [14]). The L4-L2/3 connection is thus of a low synaptic efficacy. The quantal components of the unitary EPSP as determined by mean EPSP-variance analysis by
Silver (Fig. 4b) indicated, in combination with anatomical reconstructions of cell pairs made by Lübke (Fig. 4c), that the number of anatomical synaptic contacts $\left(n_{\mathrm{a}}\right)$ on average equals the number of functional release sites $\left(n_{\mathrm{f}}\right)$, similar to the findings for the calyx of Held. An important difference is however that the intrinsic release probability at each $\mathrm{AZ}$ of the L4-to-L2/3 connection is high, of the order of $p_{\mathrm{r}}=0.8$.

Thus, a single L4-L2/3 connection, that is part of the pathway which mediates tactile stimulus representation in the supragranular layer of the somatosensory cortex, is "weak" but "reliable". This finding suggests that the sparse AP representation and the high variability in response to tactile stimuli in L2/3 are not dominated by the synaptic efficacy of individual L4-L2/3 connections. Rather, it 
a
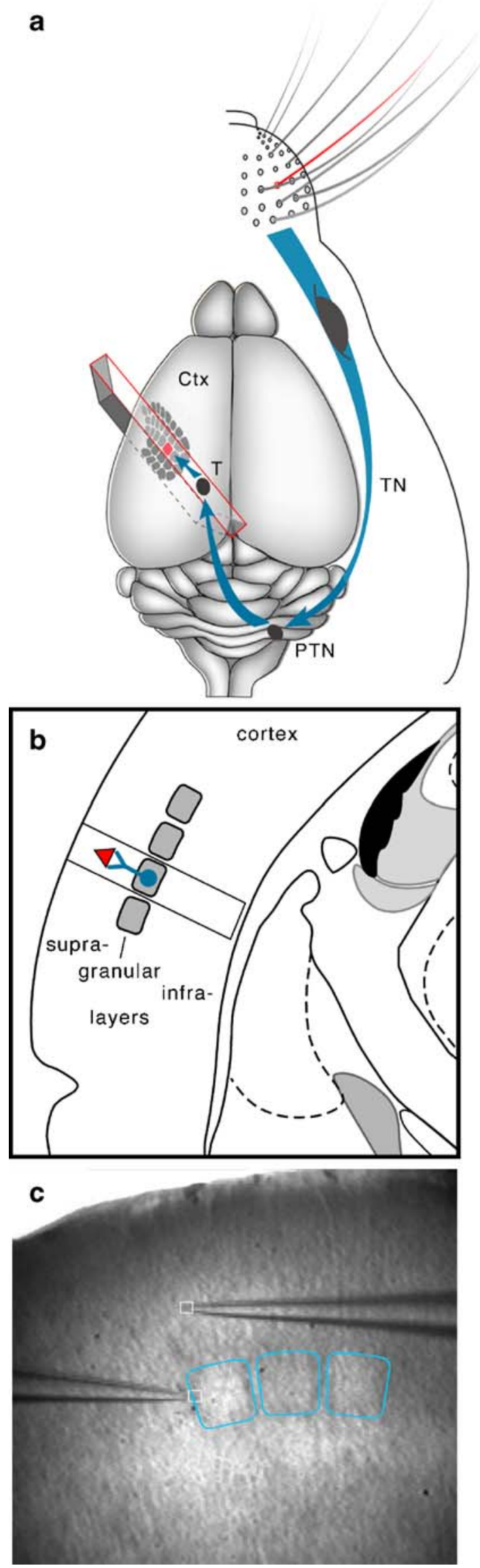

Fig. 3 Synaptic transmission between two cortical layers of the rat somatosensory system. a Schematic illustration of afferent point-to-point connection between whisker follicles at the rats' snout and a patch of cortex (red). Modified from Knott et al. [22]. TN Trigeminal nerve, $P T N$ principal nucleus of the trigeminal nerve, $T$ thalamus, $C T X$ cortex. The cutting plane for preparing acute slices of somatosensory cortex is indicated schematically for the left hemisphere. b Detailed schematic view of interlaminar cortical pathway. Granular (dark gray patches), supragranular, and infragranular layers, respectively, are indicated. Pyramid located in supragranular layer is indicated as red triangle. It is activated monosynaptically by a spiny stellate cell (blue) in granular layer. c IR-DIC view of an acute cortical slice in vitro. Outlines of some barrels in granular layer are shown in blue. Tips of recording patch pipettes are located in granular layer (left) and supragranular layer (right) [14]

reflects a network property of the ensemble of L4 cells connecting to the ensemble of L2/3 cells such as the low synchrony of L4 cell responses and their sparseness to simple stimuli like a single whisker deflection.

Two limiting types of excitatory CNS synapses In a simplified view, derived from the neuromuscular junction [21], the synaptic efficacy (or strength of a synapse) is described by the size of the PSP evoked by a single presynaptic AP, as $\mathrm{PSP}=n_{\mathrm{f}} \cdot q \cdot p_{\mathrm{r}}$. Here, $n_{\mathrm{f}}$ is the number of vesicle release sites or AZs, $q$ is the quantal size, and $p_{\mathrm{r}}$ is the average release probability at each site. For both synapses that we examined, the estimates of $n_{\mathrm{f}}$ were close to the estimates of $n_{\mathrm{a}}$, suggesting that the single vesicle hypothesis [23] applies to these mammalian CNS synapses. Synaptic efficacy in the CNS thus can formally be described by the above relation, and it is useful to compare the efficacy of the two types of synapses and the factors determining it. Eventually, one might relate them to specific functions in their respective networks.

The AVCN-MNTB synapse in the brain stem contributes to sound localization by reliably transmitting phaselocked APs, thereby generating timed inhibition at binaurally innervated neurons of the lateral superior olive [17]. In the AVCN-MNTB pathway, the synaptic efficacy is high (average unitary EPSP, $>30 \mathrm{mV}$ ), and temporal variability of first APs is very low. The main factor for the high efficacy is the large number of synaptic contacts $(\approx 600$ AZ) in combination with an estimated quantal event size of (at rest) in the range of $\approx 500 \mu \mathrm{V}$. The release probability at each AZ $\left(p_{\mathrm{r}}=0.25-0.4\right)$ is relatively low [27]. However, because of the very short duration of the local $\left[\mathrm{Ca}^{2+}\right]_{\mathrm{i}}$ transient, the release from the different AZs of the calyx is highly synchronized during an AP, and APs evoke a rapidly rising EPSC underlying the short latency $(<1 \mathrm{~ms})$ between the EPSP onset and AP onset (Fig. 5a). Here, the $\left[\mathrm{Ca}^{2+}\right]_{\mathrm{i}}$ dependency of release is such that during a presynaptic AP, the local peak $\left[\mathrm{Ca}^{2+}\right]_{\mathrm{i}}$ rises close to the $K_{\mathrm{D}}$ of the $\mathrm{Ca}^{2+}$ 
a

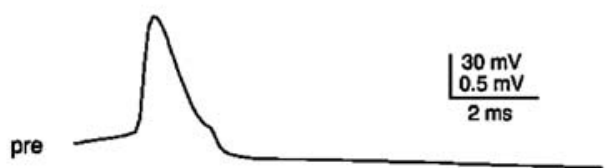

L2/3

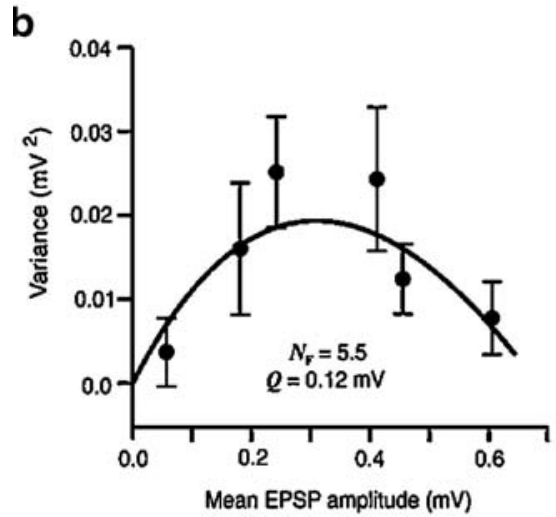

C

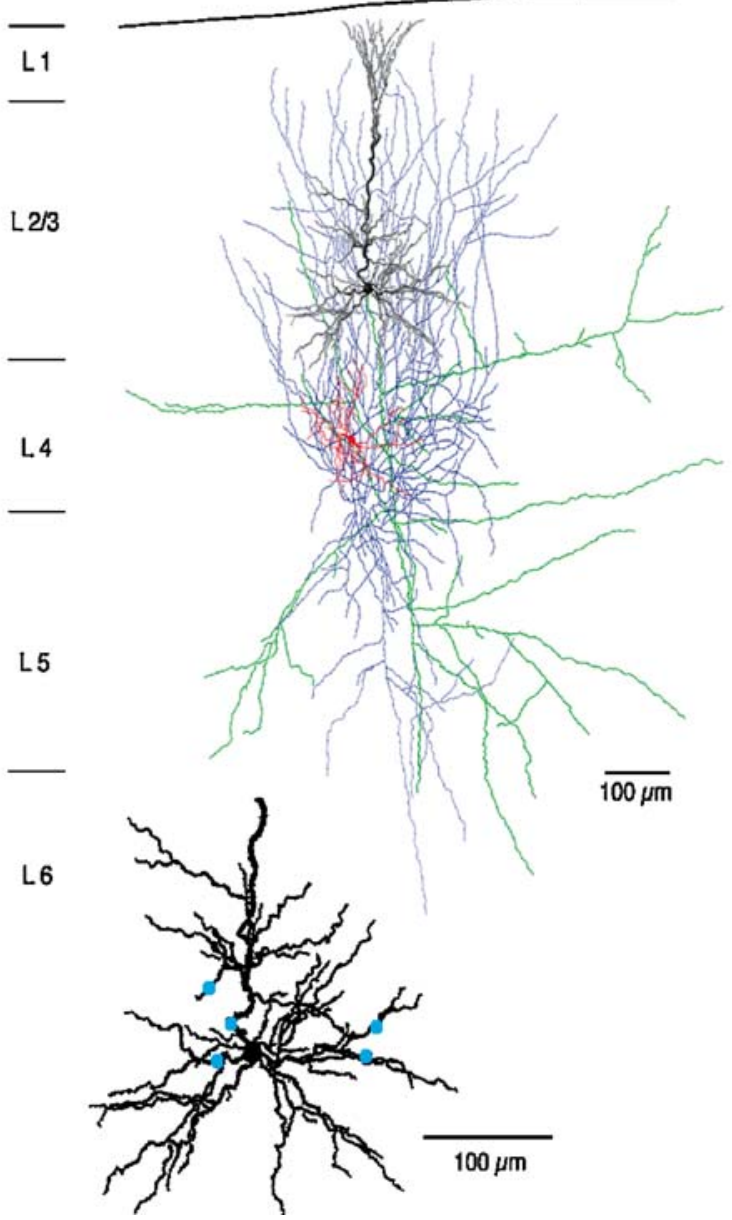

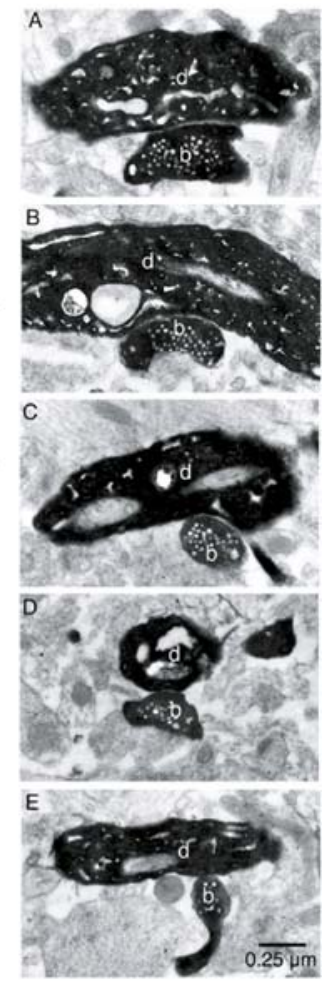

Fig. 4 Reliability of synapses in the neocortical L4-L2/3 pathway. a Simultaneous pre- and postsynaptic recording from L4 spiny stellate and L2/3 pyramidal cell. Single presynaptic AP (upper trace) evokes a unitary EPSP in a L2/3 pyramid. Several unitary EPSPs are superimposed to illustrate fluctuation in unitary EPSP peak amplitude during repeated stimulations (bottom trace, [14]). b Estimate of the number of functional synaptic contacts measured by mean EPSPvariance analysis in conditions of reduced release. In this experiment,

sensor. This means that release and transmission are sensitive to changes in the presynaptic AP wave shape [5].

The intracortical L4 spiny stellate-L2/3 pyramid synapse is, possibly, another prototypical CNS synapse. This synapse is part of the L4-L2/3 signaling pathway that transmits excitation from the recipient granular layer to the supragranular layers of cortex. The function of the pathway is to transmit excitation from the deflection of a single or multiple whisker(s) and allow integration of excitation in L2/3 cells by their lateral axon collaterals in supra- and infragranular layers. Eventually, the integrated excitation generated by deflection of whiskers is conveyed, via long-range projections from L2/3, to other cortical areas (e.g., motor cortex, association cortex, etc.). The synaptic efficacy of individual L4-L2/3 connections is low, with an average unitary EPSP of $\sim 0.6 \mathrm{mV}$. Synapses are weak because of a small number of contacts $(\approx 5 \mathrm{AZs})$
5.5 functional vesicle release sites were calculated from the shape of the parabola fitted to data points [35]. c Anatomical reconstruction of interlaminar connection between a L4 cell (red dendrites) and a L2/3 cell (black dendrites). Axon arbor of presynaptic L4 cell is in blue. Distributed location of synaptic contacts on dendritic arbor of L2/3 pyramid is illustrated in the inset by the blue dots. EM micrographs of the five anatomical contacts in this cell pair are shown on the right panels labeled $A-E$ (from Silver et al. [35])

established between L4 and L2/3 neurons and a small quantal size of $<200 \mu \mathrm{V}$, but each connection transmits reliably.

How does synaptic efficacy and variability contribute to the sensory evoked response of a population of cells and its trial-to-trial variability in a CNS pathway? In the auditory pathway, the click response of MNTB neurons are phaselocked APs. There is little difference between AVCN and MNTB cell responses, and the sensory responses in both nuclei are effective and reliable [29]. In the afferent somatosensory system, the response to a tactile stimulus in the supragranular layer L2/3 is mediated by the L4-toL2/3 pathway. Here, the response is sparse $(<1 \mathrm{AP})$ and variable for an individual $L 2 / 3$ pyramid and for the population of L2/3 pyramids [8], (de Kock et al., submitted). For comparing the possible synapse-related factors that contribute to the very different AP responses 


\section{a}

\section{AVCN (bushy cell) - MNTB (principal cell) pathway}

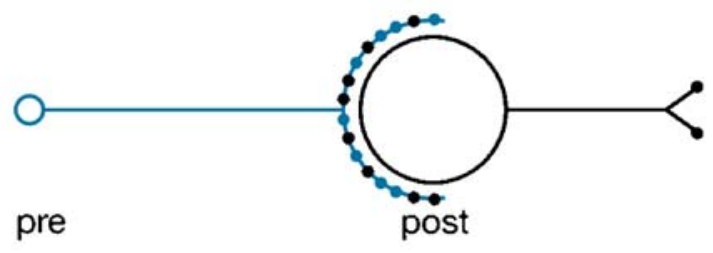

b

\section{L4 (spiny stellate cells) - L2/3 (pyramid cell) pathway}

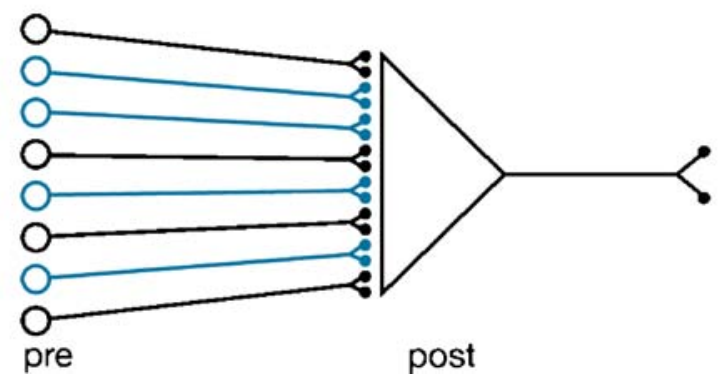

Fig. 5 Two types of synapses in CNS pathways. a A synaptic connection with high efficacy in a relay pathway of the auditory system. Convergence in this pathway is low, (1:1). A single presynaptic neuron establishes many AZs (black or blue dots) capable of releasing $n=16$ quanta of transmitter on the postsynaptic MNTB principal cell. A fraction of these AZs $(n=8)$ is activated (blue dots) by a single $\mathrm{A}$ in almost perfect synchrony following an acoustic stimulus. b A synaptic connection with low efficacy in an interlaminar pathway of the somatosensory system. Convergence in this pathway is high (8:1). A single presynaptic neuron establishes few AZs (blue or black dots) that can release two quanta. Because of the high convergence, in total, also many $(n=16)$ AZs from L4 cells (blue and black dots) are established on each postsynaptic cell. A fraction of these AZs $(n=8)$ is activated by a tactile stimulus (blue dots) but with low synchrony

to a sensory stimulus in the MNTB and in L2/3, one has to take into account the number of presynaptic neurons that converge on a postsynaptic neuron. In the AVCN-MNTB pathway, convergence is 1:1, the lower limit value (Fig. 5a). Typically, $\sim 600$ synaptic contacts are established by a single connection [33]. Of these, about $200 \mathrm{AZs}$ are active upon a sensory stimulus. In the cortex L4-L2/3 pathway, the number of contacts in an individual connection is low, $\sim 4.5$ contacts on average [24]. However, convergence between L4 spiny cells and L2/3 pyramidal cells is high, about 300-400:1 [24]. Thus, each pyramid in $L 2 / 3$ is targeted by $\sim 1,500$ AZs from L4, each of which is transmitting reliably following an AP (at least in vitro). Between 10 and $40 \%$ of L4 cells are generating APs upon a tactile stimulus [7] (de Kock et al., submitted), controlling release from $\sim 120-480$ AZs of L4 cell terminals contacting a single $\mathrm{L} 2 / 3$ cell. With this estimate of active AZs, the evoked responses in $\mathrm{L} 2 / 3$ could be tens of $\mathrm{mV}$ and reliable ( $\geq 1 \mathrm{AP} /$ stimulus). Here, however, population synchrony of APs in L4 and thus of release from L4 spiny stellate terminals is low when compared with AVCN in the brainstem pathway, where all AZs are under the control of one AP. One may therefore infer that one main source of sparse single cell responses $(<1 \mathrm{AP})$ and their higher variability in cortical L2/3 is the weaker synchrony of APs in the population of presynaptic L4 cells (Fig. 5b). Experimentally, this difference in synchrony of release in the two pathways is demonstrated by the short latency between EPSP onset and AP onset in MNTB neurons $(<0.5 \mathrm{~ms}$, Fig. 2a) and the more than tenfold longer latency between EPSP onset and AP onset in L2/3 pyramids [8].

Thus, the functional differentiation of the calyx-principal neuron synapse within the AVCN-MNTB pathway seems to underlie a well-defined function of the acoustic system, sound localization. The contribution of the spiny stellate-pyramid cell synapse to cortical representation of tactile stimuli is less clear. One may speculate that the reliable transmission of individual connections in the L4-L2/3 pathway may be a requirement for the detection of stimulus-evoked synchronous input into the cortex, for example, for the detection of the direction of whisker deflections or of their temporal order. Such population synchrony in L4 would evoke reliably larger AP responses in $L 2 / 3$. This view is supported by the experiments described in the next section, where the determination of the sensory response by the synchrony of converging inputs is illustrated for the tc projection.

In vivo pair recording from a projection synapse connecting thalamus and cortex The granular layer (L4) of somatosensory cortex which drives L2/3 is itself excited by thalamic inputs mostly from the VPM (Fig. 6a,b). Activation of cortical L4 has been examined in vitro in detail [16]. Minimal stimulation experiments in acute slices indicate that the tc synapses have a higher synaptic efficacy than intracortical synapses, similar to the L4-L2/3 type described above. They could thus be more comparable to the relay synapse in the brain stem, and cortical activation by sensory stimuli would rely on strong synapses.

One drawback inherent to the acute brain slice is the fact that in vitro, a large portion of (presynaptic) axonal arbors is removed. This prevents measurements with simultaneous pair recordings that are necessary to study the long-range connections that exist between virtually all brain regions. This shortcoming led Bruno to develop a technique [9] for pair recording in vivo to examine the properties of synapses in the connections between thalamus and cortex (Fig. 6b,c) so as to test the relative importance of synaptic efficacy and population synchrony in tactile stimulus representation. Knowing the size of a unitary EPSP in a single VPM-to-L4 


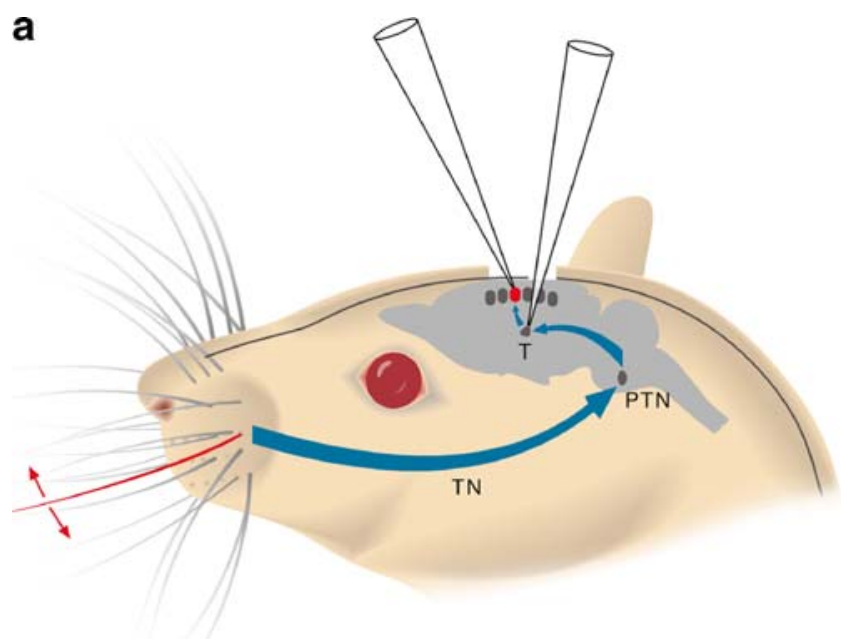

b

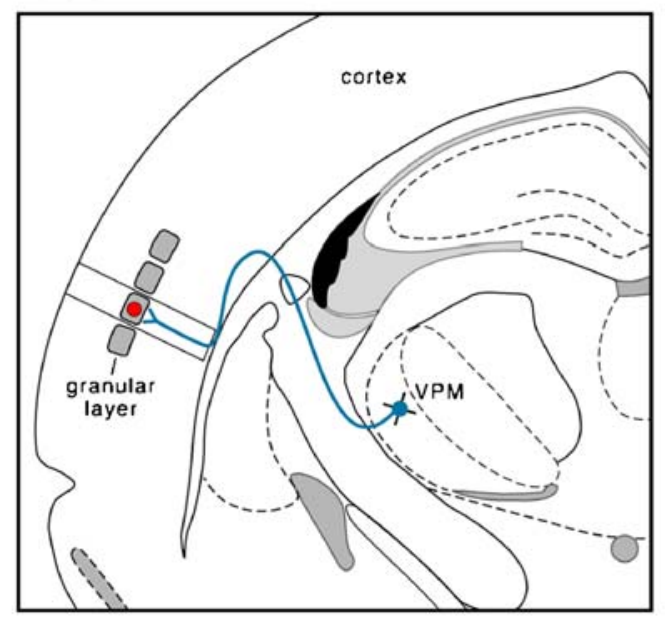

C

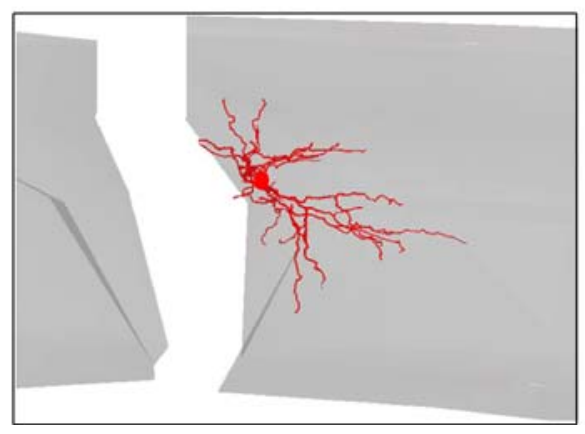

pair would allow one to set a minimum bound on how many thalamic APs mediate the compound PSP evoked in L4 spiny stellate cells by deflection of a single whisker. It constitutes a first step in delineating factors that contribute to the magnitude of stimulus representation and its trial-totrial reliability in L4.

A single AP in the VPM evoked an average unitary EPSP of about $0.5 \mathrm{mV}$ in size (Fig. 7a). Thus, under in vivo conditions, the individual VPM-L4 synapse is "weak", as the interlaminar L4-L2/3 synapse described above and the intralaminar L4-L4 synapse in vitro [13], suggesting that convergence and synchrony are the main determinants of
4 Fig. 6 Synaptic transmission in vivo between thalamus and cortex of the rat somatosensory system. a Schematic illustration of somatosensory system with afferent pathways from whisker follicles to the neocortex. TN Trigeminal nerve, PTN principal nucleus of the trigeminal nerve, $T$ thalamus. Whole-cell and juxtasomal (cellattached) recording with two patch pipettes from a barrel in L4 (red) and thalamus (gray). b Detailed schematic view of the lemniscal thalamocortical pathway (blue). Thalamic VPM cell is located $\approx 5 \mathrm{~mm}$ below the cortical surface. The cortical cell (red) is located in granular L4 of the primary somatosensory cortex. c $2 \mathrm{D}$ projection of the reconstruction of dendritic arbor of a spiny stellate cell in L4 of somatosensory cortex made after filling the cell during in vivo wc recording. Gray indicates outlines of the borders of two "barrels". White area in between indicates the "septum" separating the two barrels [9]

tactile stimulus response. The convergence in this pathway is high. During pair recording attempts, we found connections in about $40 \%$ of somatotopically aligned cells. Given a pool of about 200 VPM cells in each barreloid, this suggests that up to 90 VPM cells could be contacting a single L4 cell. To directly assess the synchrony of presynaptic APs arising from VPM, dual recordings were made from the thalamus (Fig. 7b, upper). The result showed that VPM cells are strongly synchronized by peripheral stimuli (Fig. 7b, lower). Because the sensory-evoked compound EPSP has a $15-$ to $25-\mathrm{mV}$ peak amplitude, this result suggests that at least $30-40$ axons (about $50 \%$ of the convergent axons) are active after a whisker deflection. The conclusion is that weak but presumably individually reliable VPM-L4 connections control neocortex by convergent synchronous APs in the VPM (Fig. 7c).

\section{Conclusions}

CNS synapses and cortical stimulus representation One conclusion that can be drawn from examining two different types of synapses in vitro is that in the CNS, the functional properties of synapses, as described by $n_{\mathrm{f}}, q$ and $p_{\mathrm{r}}$, vary widely. This differentiation, presumably, depends on the type of network to which the synapse belongs. It is therefore difficult, at present, to generalize the functional properties of a prototypical "CNS synapse" even if one restricts oneself to excitatory synapses with glutamate acting as a transmitter. Probably, functional properties of synapses are optimized by evolution and ontogeny within the context of their network function. Large functional changes could be the consequence of only minor changes in subcellular structure or in the composition of molecular components. A rather typical example for such subtle changes in structure that could have a large functional change would be an alteration of the kinetic properties of the $\mathrm{Ca}^{2+}$ sensor or its location in the $\mathrm{AZ}$ with respect to the sites of vesicular release. Unfortunately, CNS synapse 
Fig. 7 In vivo unitary postsynaptic potential in the thalamocortical pathway.

a Simultaneous pre- and postsynaptic recording from thalamic VPM cell and cortical L4 spiny stellate cell. Average presynaptic AP in a thalamic VPM cell recorded extracellularly in cell-attached configuration (juxtasomal recording, upper trace). Lower trace shows the averaged unitary EPSP from about 1,500 spike-triggered averages of the membrane potential of a single L4 neuron [9]. b Example AP trains from two thalamic neurons (TC1, TC2) recorded simultaneously in the same barreloid. Bottom panel Cross-correlations of spontaneous APs (left panels) and APs during whisker stimulation (right panels). Arrow Onset of whisker stimulus. Horizontal dash Baseline. Shading Difference from baseline in $\pm 20 \mathrm{~ms}$ window. c Schematic drawing of two possible mechanisms that might mediate efficient thalamocortical activation in the VPM-L4 pathway. The strong synapse model is derived from in vitro experiments. The synchrony model is derived from in vivo experiments

a
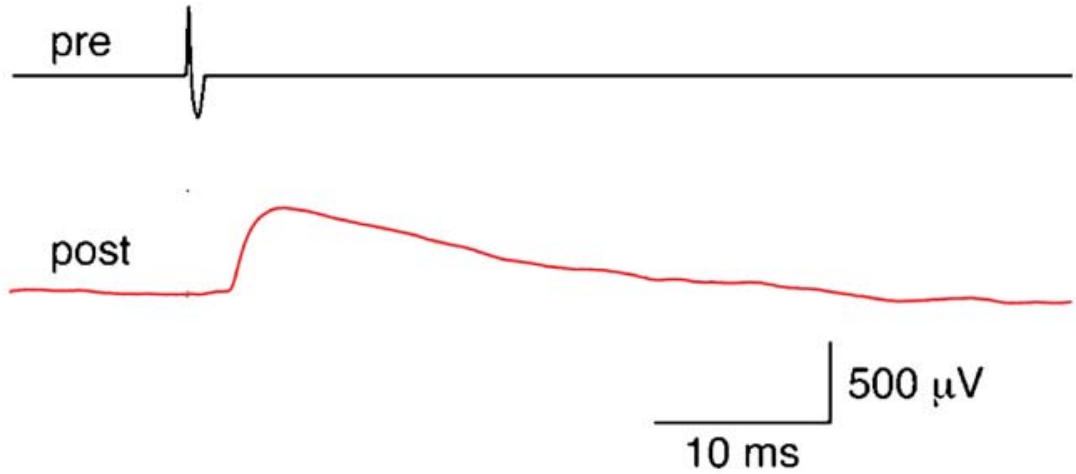

b
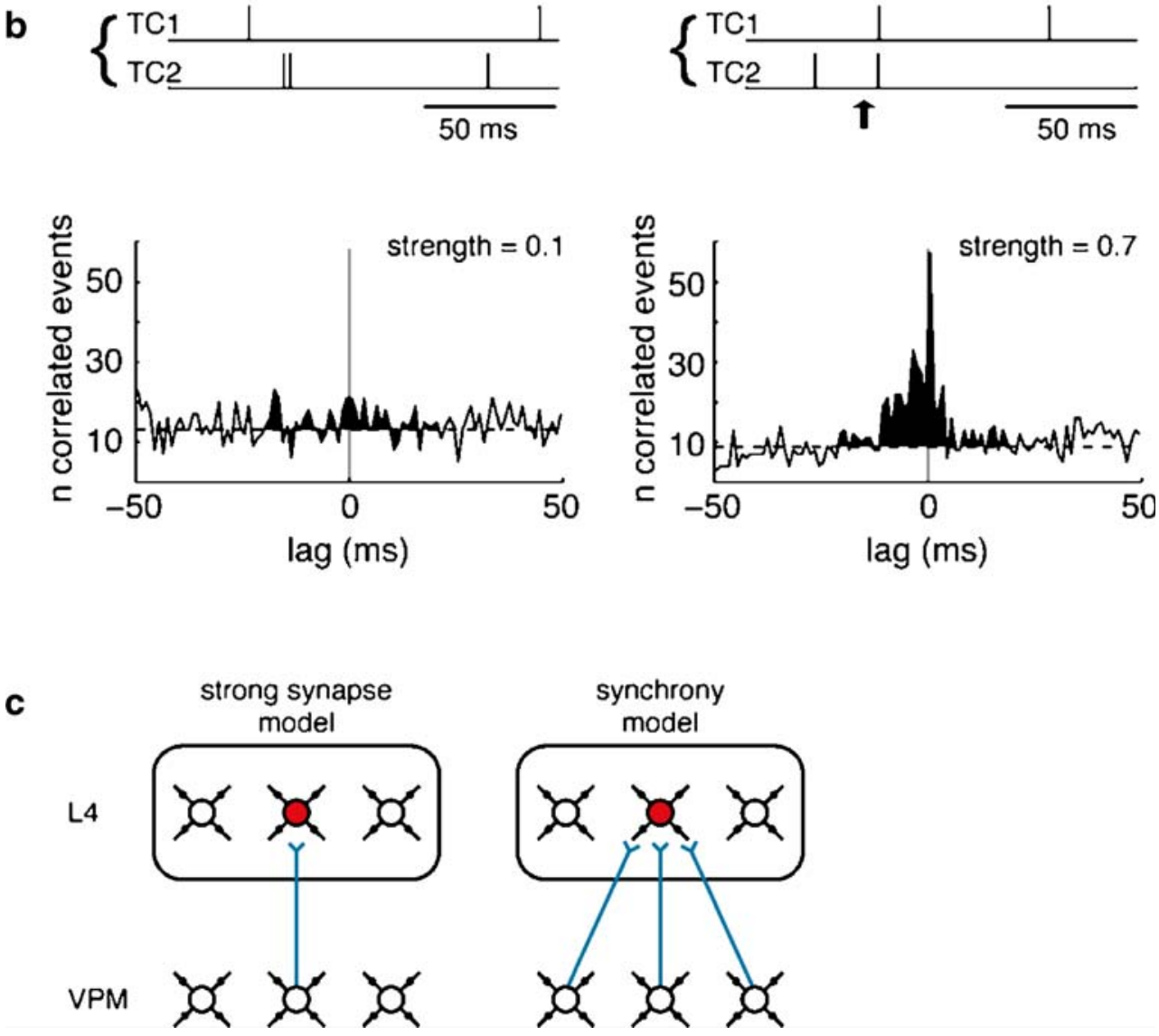

phenomena, like plasticity and target cell specification, can be understood only by first measuring in detail their biophysical properties and in addition their likely contribution to network function.

Another conclusion related to this work is that the detailed biophysical characterization of synaptic efficacy in vitro must take into account the AP patterns of pre- and postsynaptic cells in vivo to disclose the values of $n_{\mathrm{a}}, n_{\mathrm{f}}, p_{\mathrm{r}}$, and $q$ that are relevant for the understanding of CNS information processing, e.g., stimulus representation in different layers of cortex. These "effective" estimates could be very different in the intact animal, as suggested by the in vitro and in vivo experiments on the tc synapse. This seems to be particularly relevant for synapses at early stages of sensory pathways with a relatively high rate of spontaneously occurring APs and less for the intracortical pathways with their lower spontaneous AP rates.

Plasticity of synaptic efficacy in CNS synapses Unbiased recording from cortical neurons in vivo [25] indicates 
sparse response in the upper layers of somatosensory cortex at least in anesthetized animals. Obviously, induction protocols for long-term changes in CNS synapses in vitro that use high-frequency presynaptic stimulation do not seem to be adequate for mimicking the in vivo situation at least in the cortex. Dual recording in vitro also has disclosed a possible new mechanism for the induction of long-term changes in synaptic efficacy of neocortical pathways [26]. Here, the induction of mechanisms that change synaptic efficacy, which rely on coincidence of preand postsynaptic APs, may be more appropriate. One problem, however, is the low frequency of occurrence of coincident pre- and postsynaptic APs.

Column in silico project The results of investigating pathway-specific synaptic properties mean that all connections (and their synapses) have to be studied as part of their natural network in vivo. This implies also that one has to know the AP pattern that in physiological conditions is "driving" synaptic transmission in a particular pathway. An extremely useful application of patch pipettes is the configuration of Pinault [30] of "juxtasomal recording" enabling the filling of cells, revealing their exact location in a pathway and their post hoc 3D reconstruction of dendrites and axon arbors. Eventually, a set of 3D reconstructions of neurons, e.g., in different layers of a column will have to be established in conjunction with their average response properties to sensory stimuli as well as an estimate of their average connectivity. Surprisingly, most studies on cortical representation still report extracellular recordings from morphologically unidentified neurons. Given the large variation of both the AP responses and the geometry of neurons in different cortical layers and even within a layer, it seems essential for the understanding of, e.g., sensory representation to classify cell types and their respective response properties more accurately. Only then can the wide spectrum of CNS synapses be studied biophysically in the more controlled in vivo conditions with naturally occurring AP patterns. On the other hand, various models of cortical representation or "coding" could profit from more realistic assumptions made about the particular cellular properties that determine the recorded AP patterns.

Acknowledgments I would like to thank R. Bruno and E. Heil for their help in preparing this manuscript. I also thank G. Borst (Rotterdam), T. Kuner, C. de Kock, and H. Spors (Heidelberg) for the discussions, and D. Bannerman (Oxford) for the corrections in the text.

\section{References}

1. Agmon A, Connors BW (1991) Thalamocortical responses of mouse somatosensory (barrel) cortex in vitro. Neuroscience $41: 365-379$
2. Bollmann JH, Sakmann B (2005) Control of synaptic strength and timing by the release-site $\mathrm{Ca}^{2+}$ signal. Nat Neurosci 8:426-434

3. Bollmann JH, Sakmann B, Borst JG (2000) Calcium sensitivity of glutamate release in a calyx-type terminal. Science 289:953-957

4. Borst JGG, Sakmann B (1996) Calcium influx and transmitter release in a fast CNS synapse. Nature 383:431-434

5. Borst JGG, Sakmann B (1999) Effect of changes in action potential shape on calcium currents and transmitter release in a calyx-type synapse of the rat auditory brainstem. Philos Trans R Soc Lond B Biol Sci 354:347-355

6. Borst JG, Helmchen F, Sakmann B (1995) Pre- and postsynaptic whole-cell recordings in the medial nucleus of the trapezoid body of the rat. J Physiol (Lond) 489:825-840

7. Brecht M, Sakmann B (2002) Dynamic representation of whisker deflection by synaptic potentials in spiny stellate and pyramidal cells in the barrels and septa of layer 4 rat somatosensory cortex. J Physiol (Lond) 543:49-70

8. Brecht M, Roth A, Sakmann B (2003) Dynamic receptive fields of reconstructed pyramidal cells in layers 3 and 2 of rat somatosensory barrel cortex. J Physiol 553:243-265

9. Bruno R, Sakmann B (2006) Cortex is driven by weak but synchronously active thalamocortical synapses. Science 312:1622-1627

10. Chuhma N, Ohmori H (1998) Postnatal development of phaselocked high-fidelity synaptic transmission on the medial nucleus of the trapezoid body of the rat. J Neurosci 18:512-520

11. Colquhoun D, Sakmann B (1985) Fast events in single channel currents activated by acetylcholine and its analogues at the frog muscle end-plate. J Physiol 369:501-557

12. Edwards FA, Konnerth A, Sakmann B, Takahashi T (1989) A thin slice preparation for patch clamp recordings from neurones of the mammalian central nervous system. Pflügers Arch 414:600-612

13. Feldmeyer D, Egger V, Lübke J, Sakmann B (1999) Reliable synaptic connections between pairs of excitatory layer 4 neurones within a single 'barrel' of developing rat somatosensory cortex. J Physiol (Lond) 521:169-190

14. Feldmeyer D, Lübke J, Silver RA, Sakmann B (2002) Synaptic connections between layer 4 spiny neurone-layer $2 / 3$ pyramidal cell pairs in juvenile rat barrel cortex: physiology and anatomy of interlaminar signalling within a cortical column. J Physiol (Lond) 538:803-822

15. Forsythe ID (1994) Direct patch recording from identified presynaptic terminals mediating glutamatergic EPSCs in the rat CNS, in vitro. J Physiol (Lond) 479:381-387

16. Gil Z, Connors BW, Amitai Y (1999) Efficacy of thalamocortical and intracortical synaptic connections: quanta, innervation, and reliability. Neuron 23:385-397

17. Grothe B (2003) New roles for synaptic inhibition in sound localization. Nat Neurosci 4:1-11

18. Held H (1893) Die centrale Gehörleitung. Arch Anat Physiol Anat Abt 201-248

19. Helmchen F, Borst JGG, Sakmann B (1997) Calcium dynamics associated with a single action potential in a CNS presynaptic terminal. Biophys J 72:1458-1471

20. Imoto, K, Methfessel C, Sakmann B, Mishina M, Mori Y, Konno T, Fukuda K, Kurasaki M, Bujo H, Fujita Y, Numa S (1986) Location of a $\delta$-subunit region determining ion transport through the acetylcholine receptor channel. Nature 324:670-674

21. Katz B (1969) The release of neural transmitter substances. Liverpool University Press, Liverpool

22. Knott GW, Quairiaux C, Genoud C, Welker E (2002) Formation of dendritic spines with GABAergic synapses induced by whisker stimulation in adult mice. Neuron 34:265-273

23. Korn H, Triller A, Mallet A, Faber DS (1981) Fluctuating responses at a central synapse: $n$ of binomial fit predicts number of stained presynaptic boutons. Science 213:898-901 
24. Lübke J, Roth A, Feldmeyer D, Sakmann B (2003) Morphometric analysis of the columnar innervation domain of neurons connecting layer 4 and layer 2/3 of juvenile rat barrel cortex. Cereb Cortex 13:1051-1063

25. Margrie T, Brecht M, Sakmann B (2002) In vivo, low-resistance, whole-cell recordings from neurons in the anaesthesized and awake mammalian brain. Pflügers Arch 444:491-498

26. Markram H, Lübke J, Frotscher M, Sakmann B (1997) Regulation of synaptic efficacy by coincidence of postsynaptic action potentials and EPSPs. Science 275:213-215

27. Meyer AC, Neher E, Schneggenburger R (2001) Estimation of quantal size and number of functional active zones at the calyx of Held synapse by nonstationary EPSC variance analysis. J Neurosci 21:7889-7900

28. Neher E, Sakmann B (1976) Single-channel currents recorded from membrane of denervated frog muscle fibres. Nature 260:799-802

29. Paolini AG, FitzGerald JV, Burkitt AN, Clark GM (2001) Temporal processing from the auditory nerve to the medial nucleus of the trapezoid body of the rat. Hear Res 159:101-116

30. Pinault D (1996) A novel single-cell staining procedure performed in vivo under electrophysiological control: morpho-functional features of juxtacellularly labeled thalamic cells and other central neurons with biocytin or neurobiotin. J Neurosci Methods 65:113-136
31. Sahara Y, Takahashi T (2001) Quantal components of the excitatory postsynaptic currents at a rat central auditory synapse. J Physiol (Lond) 536:189-197

32. Sakmann B, Patlak J, Neher E (1980) Acetylcholine activated channels show burst kinetics in the presence of desensitizing concentrations of agonists. Nature 286:71-73

33. Sätzler K, Söhl LF, Bollmann JH, Borst JGG, Frotscher M, Sakmann B, Lübke J (2002) Three-dimensional reconstruction of a calyx of Held in its postsynaptic principal neuron in the medial nucleus of the trapezoid body. J Neurosci 22:1056710579

34. Schneggenburger R, Neher E (2000) Intracellular calcium dependence of transmitter release rates at a fast central synapse. Nature 406:889-893

35. Silver RA, Lübke J, Sakmann B, Feldmeyer D (2003) Highprobability uniquantal transmission at excitatory synapses in barrel cortex. Science 302:1981-1984

36. Stuart GJ, Dodt HU, Sakmann B (1993) Patch clamp recordings from the soma and dendrites of neurones in brain slices using infrared video microscopy. Pflügers Arch 423:511-518

37. Woolsey TA, Van der Loos H (1970) The structural organization of layer IV in the somatosensory region (S I) of mouse cerebral cortex: the description of a cortical field composed of discrete cytoarchitectonic units. Brain Res 17:205-242 